\title{
Comments to the Article "The Use of Plasma Actuators in Narrowband Active Noise Control" by A. Nasiri Published in J. Fusion Energy (2011) 30, pp. 394-397
}

\author{
Xun Huang
}

Published online: 1 March 2012

(C) Springer Science+Business Media, LLC 2012

We would like to point out that some contents (including texts and one figure) of the article "The Use of Plasma Actuators in Narrowband Active Noise Control" by A. Nasiri, published in J. Fusion Energy (2011) 30(5), pp. 394-397 are identical to the article "Design of an Active Noise Control System using Plasma Actuators" by Xun Huang, Sammie Chan, Xin Zhang and Steve Gabriel at University of Southampton in 2007. The latter manuscript is denoted by Huang07Ver0 for brevity in the following. Huang07Ver0 is actually an abstract of the conference paper (AIAA Paper 2007-3561, denoted by Huang07Ver1 in the following, with the final title of "Variable Structure Model for Flow-Induced Tonal Noise Control with Plasma Actuators") for 13th AIAA/CEAS Aeroacoustics Conference (28th AIAA Aeroacoustics Conference) at Rome, Italy in 2007. The conference paper Huang07Ver1 was further extensively modified and finally published in AIAA Journal (2008), 46(1), pp. 241-250. We would like to pay attention that Nasiri improperly borrowed contents from Huang07Ver0, which can be openly accessed at: http://www.coe.pku.edu.cn/tpic/20108231123 56675.pdf.

These coincidences, for example, are:

1. Figures 3 in Nasiri's article on pp. 395 is a copy of Figure 1 in Huang07Ver0. The caption is identical except replacing the original symbol of "_," by "solid line" and "-" by "dotted line".
2. The text in the third paragraph, right column, on pp. 394 is identical to the first paragraph in Huang07Ver0. They are: "The plasma actuator is a relatively new and promising technology for fluid and acoustic control applications due to their simplicity and lack of mechanical moving parts...The second avenue of research is to develop a closed-loop control method to attenuate flow-induced noise actively". The total word number of the exactly identical text is 139 .

Nasiri cited Huang07Ver0 in references [9] as (X. Huang, S. Chan, X. Zhang, S. Gabriel, Design of an Active Noise Control System using Plasma Actuators (2006)). However, Huang07Ver0 is an abstract but not a full length paper. The information of the citation is therefore misleading. And more importantly, the citation doses not justify the operation of identical copying of the text and figure in Huang07Ver0.

Last but not the least, the mechanisms of active noise control using plasma in Huang07Ver0 is totally different from the so-called active noise cancellation, which Nasiri presumably applied in his research. The detailed theoretical discussion is beyond the scope of this comment. Interested readers can refer to the review paper: Xun Huang and Xin Zhang, "Plasma Actuators for Noise Control", International Journal of Aeroacoustics (2010), 9(4), pp. 679-704.

X. Huang $(\bowtie)$

State Key Laboratory of Turbulence and Complex Systems, Department of Aeronautics and Astronautics, College of Engineering, Peking University, Beijing 100871, China e-mail: huangxun@pku.edu.cn 